Int. J. Dev. Biol. 60: 5-12 (2016)

doi: $10.1387 / \mathrm{ijdb} .150222 \mathrm{cl}$

\title{
Live imaging reveals spatial separation of parental chromatin until the four-cell stage in Caenorhabditis elegans embryos
}

\author{
JITKA BOLKOVÁ and CHRISTIAN LANCTÔT*,1 \\ Institute of Cellular Biology and Pathology, First Faculty of Medicine, Charles University in Prague, Prague, Czech Republic
}

\begin{abstract}
The parental genomes are initially spatially separated in each pronucleus after fertilization. Here we have used green-to-red photoconversion of Dendra2-H2B-labeled pronuclei to distinguish maternal and paternal chromatin domains and to track their spatial distribution in living Caenorhabditis elegans embryos starting shortly after fertilization. Intermingling of the parental chromatin did not occur until after the division of the AB and P1 blastomeres, at the 4-cell stage. Unexpectedly, we observed that the intermingling of chromatin did not take place during mitosis or during chromatin decondensation, but rather $\sim 3-5$ minutes into the cell cycle. Furthermore, unlike what has been observed in mammalian cells, the relative spatial positioning of chromatin domains remained largely unchanged during prometaphase in the early $C$. elegans embryo. Live imaging of photoconverted chromatin also allowed us to detect a reproducible $180^{\circ}$ rotation of the nuclei during cytokinesis of the one-cell embryo. Imaging of fluorescently-labeled P granules and polar bodies showed that the entire embryo rotates during the first cell division. To our knowledge, we report here the first live observation of the initial separation and subsequent mixing of parental chromatin domains during embryogenesis.
\end{abstract}

KEY WORDS: Caenorhabditis elegans, parental chromatin, live imaging, embryogenesis, photoconversion

\section{Introduction}

Fertilization in $C$. elegans is followed by the formation of maternal and paternal pronuclei in the zygote. The main steps in this process are the decondensation of sperm and oocyte chromatin and the addition of a nuclear envelope. These steps are preceded on the maternal side by completion of both meiotic divisions and, on the paternal one, by the substitution of the putative protamines SPCH-1,2,3 for histones in the sperm chromatin (Chu et al., 2006). Epigenetic changes have been reported to occur during formation of the pronuclei. For instance, the maternally derived histone variant $\mathrm{H} 3.3$ rapidly incorporates into both pronuclei whereas the $\mathrm{H} 2 \mathrm{~A}$ variant HTZ-1 is removed (Ooi et al., 2006; Samson et al., 2014). The paternal chromatin is hypoacetylated relative to the maternal one, especially at the H4K16 site, whereas the overall levels of histone methylation do not differ significantly between the two pronuclei (Samson et al., 2014). Once pronuclei are formed, DNA replication is initiated and the pronuclei start their migration toward each other (Edgar and McGhee, 1988). After meeting of the pronuclei, the first embryonic mitotic division takes place, quickly followed by subsequent divisions of embryonic blastomeres in a reproducible and determined manner (Deppe et al., 1978; Sulston et al., 1983).

Previous work, mainly done in the mouse embryo, sought to determine the timing and the extent of intermingling of the parental genomes after their initial apposition in the zygote. Ito and colleagues treated male mice with the halogenated thymidine analog bromo-deoxyuridine (BrdU) in order to label sperm DNA (Ito et al., 1988). Mating with untreated females allowed them to distinguish parental genomes in the resulting embryos. These authors found no obvious pattern in the positioning nor in the distribution of paternal DNA strands, but they mention that their results cannot exclude patterned segregation during the first few embryonic cell divisions. In a more recent and detailed study, and using a similar approach of labeling sperm DNA with BrdU,

Abbreviations used in this paper: BrdU, bromodeoxyuridine; FISH, fluorescence in situ hybridization, MosSCI, Mos1-mediated single copy insertion.

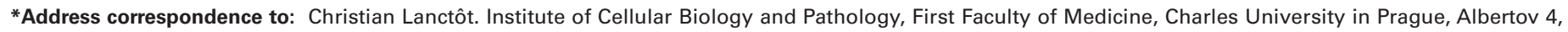
12800 Prague, Czech Republic. Tel. +420-224-968-015. Fax. +420-224-917-418. E-mail: christian.Ianctot@If1.cuni.cz; www.lanctotlab.org
}

1 Present address: BIOCEV- Department of Cell Biology, Faculty of Science, Charles University in Prague, Průmyslová 595, 25242 Vestec, Czech Republic.

E-mail : lanctotc@natur.cuni.cz

Supplementary Material (3 videos, 2 figures, 1 table) for this paper is available at: http://dx.doi.org/10.1387/ijdb.150222cl

Accepted: 24 September 2015

ISSN: Online 1696-3547, Print 0214-6282

(c) 2016 UPV/EHU Press

Printed in Spain 
Mayer and colleagues concluded that the parental DNA remained clearly segregated until the four-cell stage in the mouse embryo (Mayer et al., 2000b). These results were confirmed using DNA FISH to analyze the spatial distribution of the distinct centromeric sequences from each species in hybrid embryos. Interestingly, the DNA in the mouse male pronucleus was found to be actively demethylated within a few hours of fertilization, unlike what was observed in the female pronucleus (Mayer et al., 2000a; Oswald et al., 2000; Santos et al., 2002). It has been suggested that this epigenetic asymmetry is related to the spatial segregation of the parental genomes.

In C. elegans, a BrdU labeling scheme was used to detect parental DNA during embryonic development (Ito and McGhee, 1987). However, in this work the emphasis was put on determining whether the labeled DNA strands co-segregated reproducibly to the same embryonic cells (which they do not); no attempt was made to investigate the mixing of paternal and maternal chromatin after fertilization. We therefore decided to study this process in the living $C$. elegans embryo. We found that the maternally- and paternally-derived chromatin did not mix until after the division of the $\mathrm{AB}$ and $\mathrm{P} 1$ blastomeres, in the 4-cell embryo. To our knowledge, we report here the first live observation of the initial separation and subsequent mixing of the parental chromatin in an early embryo.

\section{Results}

\section{Generation and characterization of a C. elegans strain expressing a photoconvertible form of histone $H 2 B$ in the germline and in early embryos}

To be able to distinguish the parental genomes in the early $C$. elegans embryo, we first tried to specifically label the maternal DNA by injecting a fluorescent derivative of a nucleotide (Cy3-dUTP) into the adult gonad. In doing so, we had hoped that the label would be incorporated in the replicating DNA in the distal gonad and that the labeled DNA would eventually have ended up in the maternal pronucleus after meiosis. Although labeling of the DNA in the gonad and in the oocytes proved to be easy and efficient, we failed to obtain any significant number of early embryos in which labeled DNA could be detected, and those that did show labeled
TABLE 1

\section{PROPORTION OF CASES IN WHICH \\ THE PATERNALLY- AND MATERNALLY-DERIVED CHROMATIN WERE OBSERVED TO BE SPATIALLY SEPARATED}

\begin{tabular}{cccccccc} 
& P0 & AB & P1 & ABa & ABp & EMS & P2 \\
\hline Prophase & $22 / 22$ & $11 / 11$ & $9 / 10$ & $0 / 7$ & $0 / 6$ & $0 / 11$ & $0 / 8$ \\
Metaphase & $26 / 26$ & $7 / 10$ & $10 / 12$ & $0 / 6$ & $0 / 3$ & $0 / 7$ & $0 / 6$ \\
Anaphase & $24 / 24$ & $8 / 11$ & $8 / 10$ & $0 / 7$ & $0 / 7$ & $0 / 6$ & $0 / 6$ \\
\hline
\end{tabular}

The number of images with separated parental chromatin / total number of images is indicated. The imaging data consisted of movies and snapshots taken at various times after photoconversion of either of the pronuclei. Results are presented according to the mitotic phase (rows) and blastomere identity (columns).

chromosomes looked abnormal (data not shown). The cause for this remains unclear, but we suspect that it might be due to the inability of Cy3-dUTP-containing maternal DNA to efficiently participate in early developmental processes. We therefore resorted to an alternative strategy to image the parental chromatin in $C$. elegans, one that is based on the expression of a photoconvertible form of histone in the germ line and on the selective photoconversion of either oocyte-derived or sperm-derived chromatin shortly after fertilization. We chose Dendra2 as a photoconvertible marker. Dendra2 is a monomeric green fluorescent protein (emission $\lambda_{\max }=$ $507 \mathrm{~nm}$ ) that can be converted to a red fluorescent protein (emission $\lambda_{\max }=573 \mathrm{~nm}$ ) upon illumination with $405 \mathrm{~nm}$ light (Gurskaya et al., 2006). Dendra2 protein efficiently matures at $20^{\circ} \mathrm{C}$, which makes it suitable for use in C. elegans. A strain expressing a Dendra2::H2B coding sequence under the control of the mex-5 promoter $(-472$ to +1 ) and fused to the $t b b-2$ ( $\beta$-tubulin) 3' untranslated region was obtained through insertion of the transgene at the ttTi5605 site using the Mos1-mediated single copy insertion (MosSCl) method (Zeiser et al., 2011).

Transgenic worms (strain JBL1) were imaged by selective plane illumination microscopy, which revealed the expected pattern of expression of the Dendra2-H2B fusion protein, i.e throughout the gonads (with stronger expression starting in pachytene nuclei) as well as in spermatozoa and in nuclei of the embryos in the uterus (Suppl.Video 1). Dendra2-H2B was detected in nuclei up to the L1 stage (data not shown). By illuminating a region-of-interest with

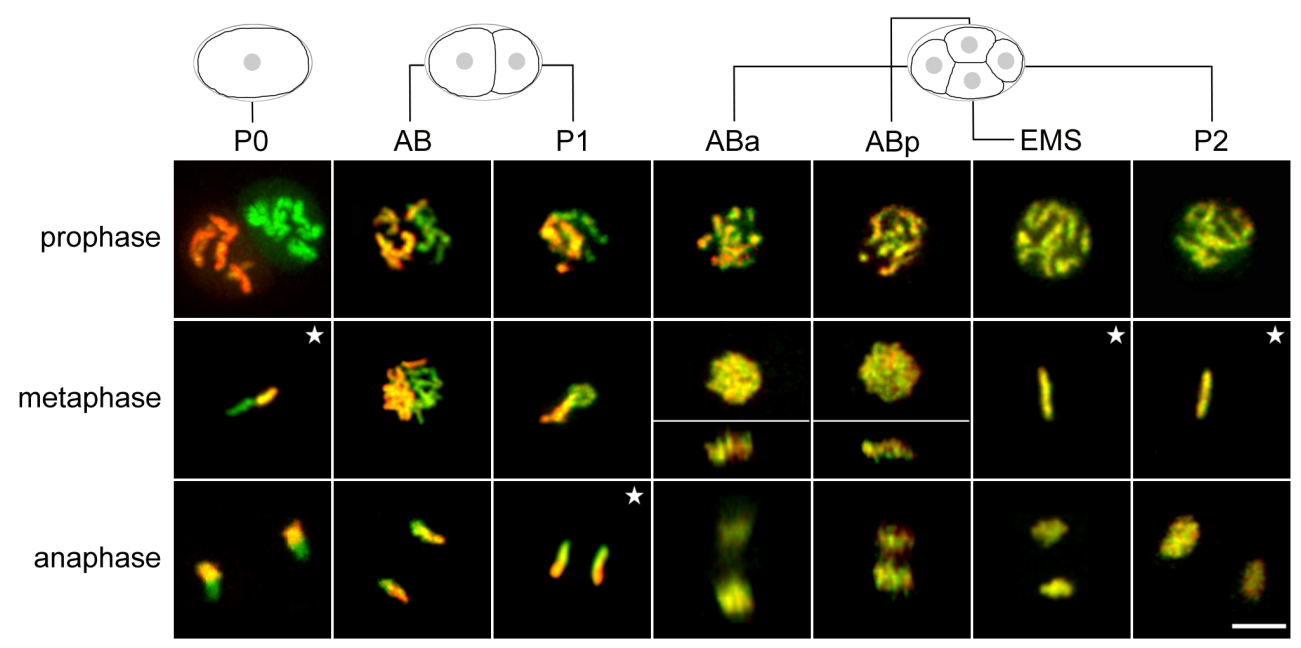

Fig. 1. Paternally- and maternally-derived chromatin remain spatially separated until the 4-cell stage. One of the pronuclei was photoconverted in the early zygote of the Dendra2-H2B-expressing strain, after which imaging of the chromatin from the photoconverted pronucleus (red) and from the otherpronucleus (green) was performed until the 4-cellstage. Representative images are taken from different embryos. Shown are maximum projections of image stacks, except for panels labeled with asterisks, which show single optical sections. Nuclei are in mitotic prophase (upper panels), metaphase (middle panels) or anaphase (lower panels). The blastomere identity is indicated and schematized on top of the corresponding panels. Note the clear separation of parental chromatin in the anaphase of $A B$ and $P 1$ and the mixing of chromatin (yellow) in all mitotic nuclei of the 4-cell embryo. Orthogonal views are shown for the ABa and ABp nuclei in metaphase and anaphase. Scale bar, $5 \mu \mathrm{m}$. 
the $405 \mathrm{~nm}$ diode laser (see Materials and Methods), a green-tored photoconversion of the labeled chromatin was observed (Fig. $\mathrm{S} 1)$. The increase in red fluorescence after photoconversion was on the order of $\sim 100$-fold (not shown). However, we noted that the green fluorescence intensity was often unchanged or only partly decreased after photoconversion. Our attempts to reach a higher proportion of photoconverted molecules (i.e. a larger decrease in green fluorescence) by increasing laser power only led to overall bleaching of both signals. It should be noted that the residual green fluorescence we observed after photoconversion did not hinder our ability to perform the analyses that are described below. Actually, the biggest problem we faced while imaging Dendra2H2B-expressing embryos was the high photosensitivity of the fluorescent fusion protein.

\section{The paternal and maternal chromatin remain separated until} after the division of the $A B$ and $P 1$ blastomeres

The strategy we used to follow the spatial distribution of parental chromatin in the early embryo was to specifically photoconvert the chromatin of either the paternal or maternal pronucleus at a time when they were still well separated in the zygote. The maternal pronucleus was identified as the closest to the polar bodies. To assess the phototoxicity of the $405 \mathrm{~nm}$ laser line, we first subjected young embryos (<50-cell stage) to our photoconversion protocol and tested whether they could develop into larvae. Out of 23 that were treated, 22 proceeded to hatch normally. To investigate the issue of phototoxicity in the zygote, we photoconverted the chromatin in one of the pronuclei and imaged the subsequent steps of embryogenesis. The time at which the metaphase plate was observed in the 1-cell embryo was taken as a reference point. The time to the next metaphase was measured for each blastomere. Although there was a trend toward a slight delay in cell division after photoconversion, i.e. $14.0 \pm 1.0$ minutes for the $A B$ cell in irradiated embryos vs. $13.1 \pm 0.4$ minutes in control embryos, the differences were not highly significant $(p=0.0681$, Mann-Whitney test, Supplementary Table 1). Taken together, our results show

Fig. 2. Spatial redistribution of chromatin occurs shortly after cell division. Half of the chromatin in the nuclei of $P 1$ was photoconverted during metaphase and subsequently imaged until the next metaphase, which was taken as a reference time point (0:00). The strain that was used (JBL2) expresses Dendra2-H2B and $\gamma$-tubulin-GFP to label both chromatin (green and red) and centrosomes (green). (A) After photoconversion, the chromatin domains remain spatially segregated throughout the remainder of mitosis (e.g. time-22:00), during chromatin decondensation (e.g. time -20:00) and during the first minutes of the next cell cycle (time-15:15). The intermingling of the chromatin domains occurs relatively abruptly (at time -13:15 in the image sequence that is shown). Shown are maximum projections corresponding to cross-sections of the embryonic nuclei of 1.5-5 $\mathrm{mm}$. Time is indicated in minutes:seconds. Scale bar, $5 \mu \mathrm{m}$. (B) Starting after the completion of the P1 division, the spatial distribution of the differently-labeled chromatin domains was assessed in the P2 nucleus at intervals of 0.5 to 2 minutes and scored either as spatially segregated or mixed. The score is plotted against the time relative to the P2 metaphase (on the $x$-axis, 0:00 represents the occurrence of this metaphase) as well as the actual cell cycle progression indicated by the distance between the centrosomes (on the $y$-axis). The spatially segregated phenotypes (black portion of the plots, $n=8$ ) are observed at the beginning of the cell cycle, but not later than 5 minutes into the cell cycle. The chromatin domains are clearly mixed (gray portion of the plots) when the two centrosomes begin to rapidly move away from each other (time point -6:00). minimal phototoxicity during photoconversion of the Dendra2-H2Bexpressing worm embryos.

The efficiency of photoconversion was similar for male and female pronuclei, and the results were similar irrespective of which pronuclear chromatin was photoconverted. After photoconversion, imaging of green and red fluorescence was performed at high speed and in three dimensions on a confocal spinning disk microscope. Similar results were observed in interphase and mitotic embryonic nuclei. However, because the accompanying condensation of chromatin led to stronger fluorescence signals, the spatial distribution of parental chromatin could be most clearly seen in mitotic nuclei (Fig.1). We observed well-defined separation of the paternal and maternal chromatin in zygotes and in two-cell embryos (Suppl. Video 2). In the latter, the chromatin was spatially segregated according to parent-of-origin in both the $\mathrm{AB}$ and $\mathrm{P} 1$ blastomeres. It was only after the division of these blastomeres that extensive intermingling of the parental chromatin occurred. This was most clearly seen on mitotic figures of 4-cell embryos. We did not observe any change in the relative spatial distribution of the parental chromatin domains during the rotation of the P1 blastomere.

The spatial distributions of parental chromatin in early blastomeres, as observed in a total of 38 movies, are summarized in Table 1. Except for cases where the Dendra2-H2B signal was too weak to conclude without any doubt, the maternal and paternal chromatin were observed to be spatially segregated in the zygote

A
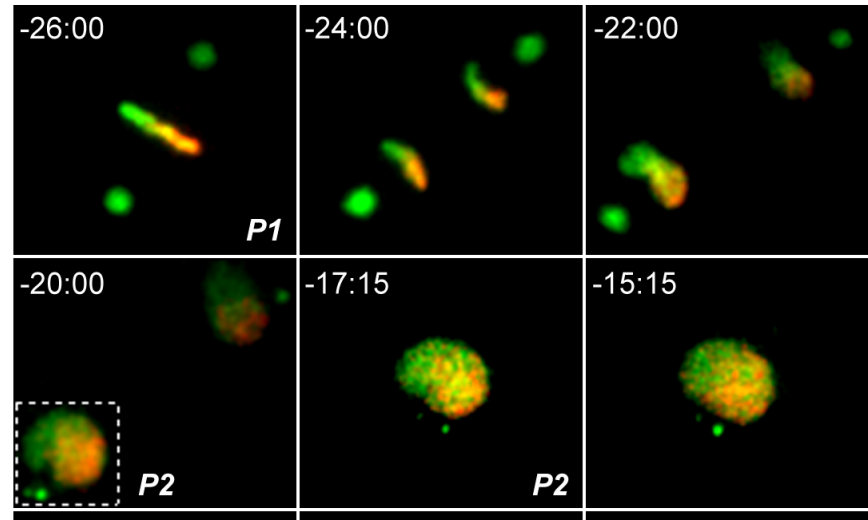

$-15: 15$
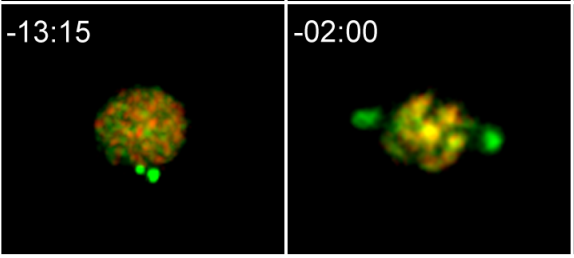

.
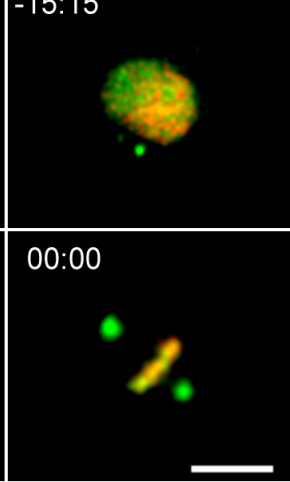

B

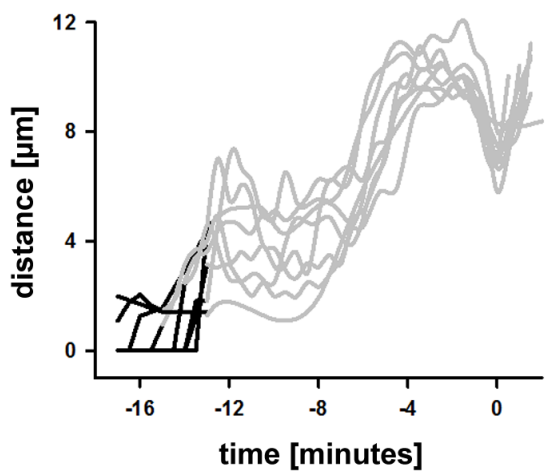



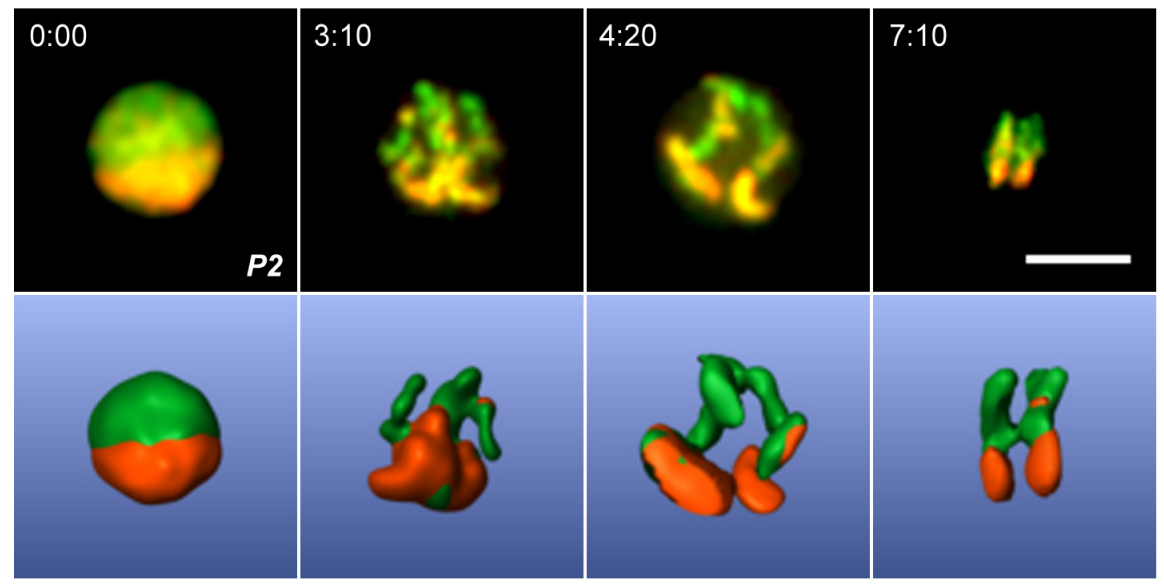

Fig. 3. The relative spatial distribution of chromatin is largely maintained during mitosis. The chromatin in half of the $P 2$ nucleus of a strain expressing Dendra2$H 2 B$ (JBL 1) was photoconverted during $S$ phase (time 0:00). Image stacks were acquired during the subsequent mitosis. Shown are representative maximum projections of image stacks acquired from prophase to anaphase (top panels) as well as the corresponding 3D reconstructions (bottom panels). Note that the spatial distribution of the photoconverted chromatin is largely maintained during mitosis (compare first and last time points). Time is indicated in minutes:seconds. Scale bar, 5 um.

(34/34 cases) and in the 2-cell embryo (12/14 cases), but never at the late 4 -cell stage ( $0 / 14$ cases). It could be argued that the intermingling we observed is not the result of chromatin movement, but rather of the exchange of histone $\mathrm{H} 2 \mathrm{~B}$ molecules between the photoconverted and the non-photoconverted chromatin domains. We believe this to be unlikely because the half-time for histone $\mathrm{H} 2 \mathrm{~B}$ exchange is on the order of hundreds of minutes (Kimura and Cook, 2001), i.e. much longer than the entire sequence of events that are imaged in the present work.

To determine whether one of the parental chromatin domains adopted a reproducible position relative to the future dorsoventral axis, either the maternal or paternal pronucleus was photoconverted and development was then imaged up to the 4-cell stage, at which point the dorsoventral orientation of the embryo could be determined from the position of the $A B p$ cell on the dorsal side (Singh and Pohl, 2014). Looking back in time, the position of the labeled chromatin domain was determined in the same embryo at the 2-cell stage. No obvious bias was found in the positioning of the parental chromatin domains along the future dorsoventral axis.

The mixing of spatially separated chromatin occurs shortly after chromatin decondensation within the first minutes of the cell cycle at the 4-cell stage

Our efforts to determine more precisely the time at which the mixing of parental chromatin occurred proved unsuccessful because the progressive loss of signal intensity from the photoconverted Dendra2-H2B molecules prevented us from distinguishing unambiguously the parental chromatin signals during the interphase of 4-cell embryos. This loss in signal intensity was likely due to a combination of photobleaching and dilution through cell division.
To counter this technical limitation, we decided to photoconvert half of the metaphase plate of a dividing blastomere of the 2-cell embryo, thus recreating the segregated chromatin pattern that had been observed in previous experiments. We then followed the respective movement of photoconverted and non-photoconverted chromatin at high temporal resolution. These experiments were carried out in the P1 cell since this blastomere divides more or less parallel to the imaging plane, which allows for higher resolution than if the cell divides along the $z$ axis, as does the AB cell. As shown on Fig. 2A, we did not observe any obvious change in chromatin distribution during chromatin decondensation after division of P1. In fact, the chromatin that had been photoconverted in half of the P1 metaphase plate remained mainly confined to half of the daughter nucleus within the first minutes after cell division. After that time, the photoconverted chromatin intermingled rather rapidly with the non-photoconverted chromatin. The mixing of the differently labeled chromatin domains was clearly observed in the subsequent mitotic stages.

To quantify these observations and to determine more precisely the timing of chromatin intermingling, we took advantage of the fact that the strain that was used expressed both Dendra2-H2B and $\gamma$-tubulin-GFP, a marker of the centrosomes. The number and position of the centrosomes was used to assess the actual stage of the cell cycle in the daughter P2 cell. Since the start of image acquisition was not precisely synchronized after the photoconversion of half of the P1 metaphase, the occurrence of the subsequent metaphase was used as a reference time point. A total of 10 movies were made, 2 of which could not be used either because of high photobleaching or because of aborted cell cycle. Images from the others $(n=8)$ were analyzed and the degree of mixing was
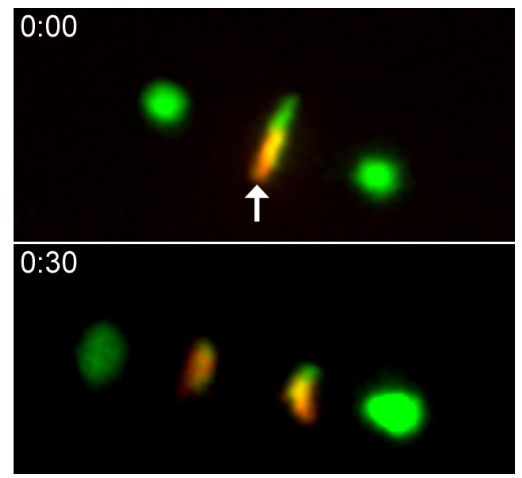

Fig. 4. The chromatin rotates by $180^{\circ}$ during the division of the one-cell embryo. The male pronucleus was photoconverted shortly after fertilization in the JBL2 strain, which expresses Dendra2$H 2 B$ and $\gamma$-tubulin-GFP to label both chromatin (green and red) and centrosomes (green). Imaging began at metaphase shortly after pronuclear fusion (time 0:00). Starting in late anaphase and continuing during telophase (time points 0:30 and 1:00), the nascent nuclei rotate by $180^{\circ}$, such that the photoconverted chromatin is found at the 2-cell stage on the opposite side relative to its initial position (compare the direction of the arrows at the first and last time points). Shown are maximum projections corresponding to a cross-section of the embryo of 6.5-10 $\mu \mathrm{m}$. Time is indicated in minutes:seconds. Scale bar, $5 \mu \mathrm{m}$. 


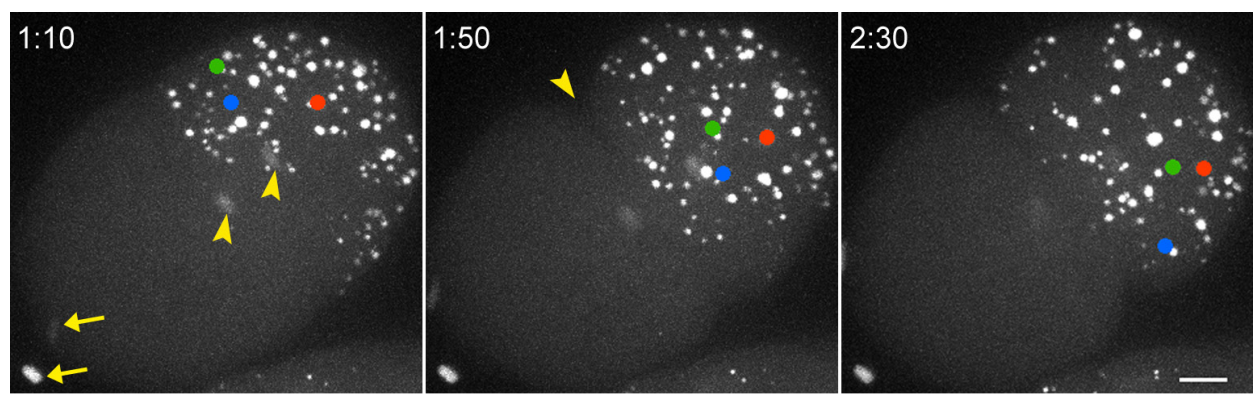

Fig. 5. The cytoplasmic $P$ granules rotate during cytokinesis of the $C$. elegans zygote. A strain expressing Dendra2-H2B and GFP-labeled PGL-1, a marker of $P$ granules, was imaged at 10-second intervals. Shown are maximum projections of image stacks of part of the embryo $(20 \mu \mathrm{m})$ at 3 different time points. Colored dots mark three individual $P$ granules, which are clearly seen to rotate around the anterior-posterior axis during cell division. In the left panel, the Dendra2-labeled chromatin in the nascent nuclei (arrowheads) and in the polar bodies (arrows) is indicated. The arrowhead in the middle panel (time 1:50) labels the asymmetrically-forming cytokinetic furrow. Time is indicated in minutes:seconds after the start of imaging. Scale bar, $5 \mu \mathrm{m}$.

plotted relative to the actual time and to the distances between centrosomes (Fig. 2B). The first occurrence of mixing occurred $4 \pm 1$ minutes after the beginning of the cell cycle, $2 \pm 1$ minutes after the appearance of two distinct centrosomes, presumably at the beginning of $S$ phase, and $6 \pm 1$ minutes before one of the centrosomes started to migrate toward the opposite pole upon mitotic entry. Taken together, these observations indicate that the intermingling of chromatin in the early $C$. elegans embryo does not occur during chromatin decondensation at the end of mitosis, but relatively abruptly 3-5 minutes into the cell cycle, well before one of the duplicated centrosomes begins to migrate toward the opposite side of the nucleus.

\section{The relative spatial distribution of chromatin is largely main-} tained throughout mitosis in the early $C$. elegans embryo

The preceding results did not exclude the possibility that the mixing of chromatin domains might occur prior to formation of the metaphase plate, as was previously observed in mammalian cells (Strickfaden et al., 2010). To investigate this issue in the early $C$. elegans embryo, the chromatin in half of a nucleus at the 2- or 4-cell stage was photoconverted during $S$ phase and chromatin distribution was followed throughout the subsequent mitosis. As shown on Fig. 3, no drastic change in relative chromatin distribution was observed at any time during mitosis. This behavior was observed in 8 out of 11 experiments.

\section{Chromatin rotates around the anterior-posterior axis during the first embryonic division}

In the course of this study, we noticed a reproducible rotation of the one-cell embryo chromatin around the anterior-posterior axis (Fig. 4 and Suppl. Video 2). The observation was made on 16 out of 18 embryos that were analyzed. We were able to detect this intriguing movement thanks to the property of Dendra2, which allowed us to visualize a reproducible spatial pattern on the metaphase plate of the one-cell embryo as a consequence of the earlier photoconversion of one of the pronuclei. The rotation of the chromatin, which was not observed at later stages, started in anaphase of the one-cell embryo and was completed by late telophase. The orientation of the rotation was right-handed around the anterior-posterior axis, meaning that if the thumb is pointing toward the posterior, the fingers point the direction of rotation. The handedness of the rotation did not depend on which of the pronuclei was photoconverted. The rotation described half a turn $\left(180^{\circ}\right)$ and was completed within about 1 minute.

To assess whether the rotational movement that we observed was limited to the nascent nuclei or whether it concerned other embryonic structures as well, we imaged the behavior of the cytoplasmic $\mathrm{P}$ granules at the time of chromatin rotation. These were labeled with a GFP-PGL-1 fluorescent fusion protein (Gallo et al., 2010). Images were acquired in $3 D$ at 10 -second intervals from metaphase until the one-cell embryo had divided. We observed a clear rotation of the P granules (in 8 out 8 cases, Fig. 5 and Suppl. Video 3). Like the chromatin, the $\mathrm{P}$ granules were found to rotate in a right-handed direction on the anterior-posterior axis and with an apparent angle of about $180^{\circ}$. The duration of rotation was longer for $P$ granules ( $\sim 3$ minutes) than for the chromatin ( 1 minute). The tracking of Dendra2-H2B-positive polar bodies provided additional information on movement of the embryo. The first polar body did not move. However, the second polar body, which unlike the first one is attached to the plasma membrane (Benenati et al., 2009), underwent an obvious rotation. Taken together, these results suggest that the entire one-cell embryo rotates inside the egg shell during cytokinesis. Importantly, we observed that the initiation of this rotational movement coincided with the asymmetric invagination of the cleavage furrow (on the presumptive left side of the embryo).

\section{Discussion}

Using photoconversion of Dendra2-labeled histone H2B in either pronuclei of the $C$. elegans zygote, we have been able to observe, to the best of our knowledge for the first time, the mixing of parental chromatin in living embryos. A previous work which used BrdU labeling of sperm DNA and immunodetection on fixed samples to follow the paternal genome throughout embryogenesis found that the parental DNA strands segregate randomly during development, but did not address the issue of genome mixing in the early embryo (Ito and McGhee, 1987). We now report that the parental chromatin remain spatially separated until after the second division of embryonic blastomeres, i.e. until the 4-cell stage. In separate experiments, we unexpectedly found that the intermingling of chromatin domains does not take place during mitosis as previously reported in cultured mammalian cells (Strickfaden et al., 2010) or during chromatin decondensation at the end of telophase. Rather, live imaging of chromatin domains through an entire cell cycle in the P1 and P2 blastomeres revealed that mixing occurs $\sim 3-5$ minutes into the cell cycle. Despite the variability we observed, it is clear from our results that 1) the chromatin domains remain spatially segregated until the appearance of two distinct centrosomes, presumably at the beginning of $S$ phase and 2) the chromatin domains have completely mixed well before the start of the rapid movement of one of the centrosomes upon mitotic entry. The results reported here are schematized on Fig. 6. 
Our observations raise a number of questions, in particular as to why the mixing of parental chromatin does not occur earlier. With regard to the zygote, the answer is simple: the contents of the pronuclei are prevented from mixing by a nuclear envelope that stays intact until the formation and alignment of individual metaphase plates in each of them (Gorjánácz and Mattaj, 2009). The rapid and directed movements of chromosomes during the subsequent anaphase are not expected to be compatible with chromatin mixing. It is not clear, however, why the parental chromatin compartments remain segregated in the $A B$ and $P 1$ blastomeres once zygotic division has been completed. It could be that an active mechanism prevents chromatin mixing in the 2-cell embryo, possibly based on epigenetic modifications. However, although there have been reports of differences in post-translational modifications of histones between the paternal and the maternal pronuclei, in particular the lack of H3K79me3 and H4K16Ac in paternal chromatin, these tend to be rapidly equalized, such that
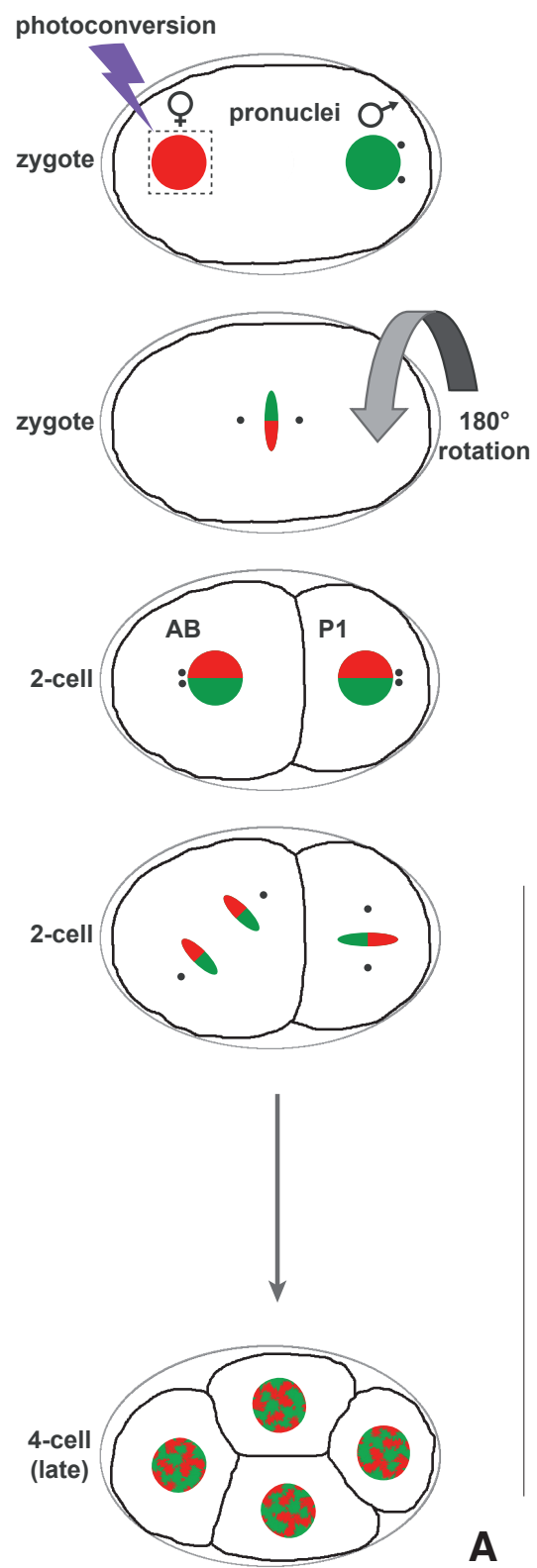

A
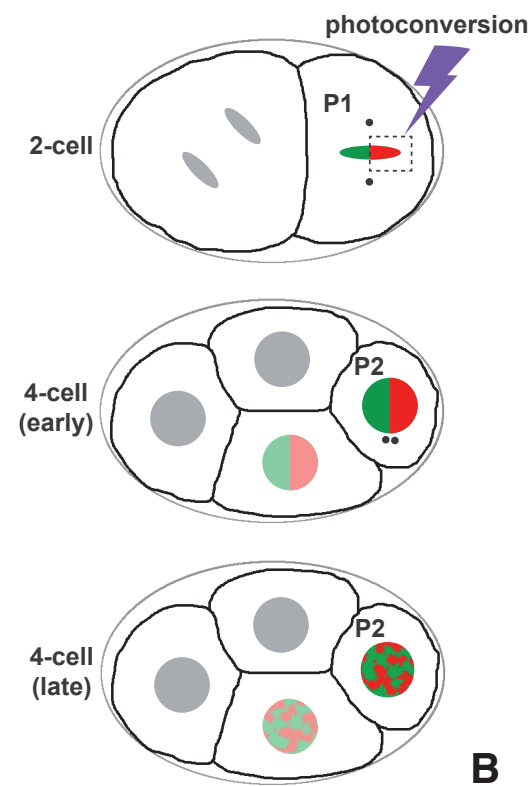

B

parental-specific histone modifications have yet to be observed at the 2-cell stage (Samson et al., 2014). Whatever the molecular mechanisms that are at work, it seems clear that a temporal window has to open in order for parental chromatin mixing to occur. Since chromatin mobility has been found in mammalian cells to be constrained during $S$ phase (Walter et al., 2003) and since we show here that, to a large extent, the spatial arrangement of chromatin is maintained during mitosis in the early $C$. elegans embryo, then we hypothesize that in this organism the length of time that separates chromatin decondensation after mitosis from the initiation of S phase (i.e. the G1 phase) is a key determinant in the process of parental chromatin mixing. In this regard, it is worth noting that a progressive lengthening of the cell cycle, which affects all lineages, has been observed during the embryogenesis of $C$. elegans (Bao et al., 2008). Although it is generally thought that this lengthening is solely due to longer $S$ phases in the early embryo, with gap phases only appearing at later time points (Kipreos, 2005), the initiation of DNA replication has not yet been imaged in the living 4-cell embryos and the appearance of a brief G1 phase at this stage cannot be excluded. We therefore suggest that it is during or shortly after this brief putative G1 phase that the spatial intermingling of parental chromatin takes place inside the cell nucleus. This interpretation is consistent with previous results which showed that chromatin mobility in mammalian cells is higher in early G1 than in the remainder of the cell cycle (Thomson et al., 2004; Walter et al., 2003). It should be noted here, however, that the presence of a putative G1 phase may not be sufficient to account for the timing of chromatin mixing. In the mouse, the parental genomes were found to be spatially segregated in the 2-cell embryo despite the fact that the blastomeres remain in G1 for several hours at this stage (Mayer et al., 2000b). Hence, additional work is needed to elucidate the mechanism of chromatin mixing. The use of a well-characterized forward and reverse genetics model such as $C$. elegans may prove helpful in this regard.

The use of a photoconvertible form of histone H2B expressed in the germ line allowed us to make additional observations on the behavior of chromatin in the early $C$. elegans embryo. As

Fig. 6. Schematic representation of parental chromatin mixing in the early $\boldsymbol{C}$. elegans embryo. (A) The generation of a worm strain expressing Dendra2-H2B in the germline allowed us to specifically label the chromatin of either the male or female pronucleus through photoconversion. Live imaging of the subsequent development showed that the parental chromatin domains remain spatially segregated in the zygote and at the 2-cell stage. It is only at the late 4-cell stage that the parental chromatin domains are found to be intermingled. Photoconversion of pronuclear chromatin also enabled us to observe a reproducible $180^{\circ}$ rotation of the whole embryo during the division of the zygote. (B) To determine more precisely the timing of chromatin mixing, differently-labeled chromatin domains were generated by photoconverting half of the metaphase plate of the P1 blastomere at the 2-cell stage. Subsequent imaging revealed that the chromatin domains remained spatially separated early after division. Mixing occurred -3-5 minutes into the cell cycle and was complete before the start of migration of one of the centrosomes. For technical reasons, these imaging experiments were preferentially carried out on the P1 and P2 blastomeres. The chromatin domains are colored in green and in red. The centrosomes are shown as small black dots. 
mentioned above, unlike what has been reported in cultured mammalian cells, where extensive rearrangements of chromatin positioning were observed during prometaphase (Strickfaden et al., 2010), we did not detect a significant redistribution of labeled chromosomes relative to each other at this stage of mitosis in $C$. elegans embryos. The cause for this difference remains to be investigated. However, it is tempting to speculate that it is related to the late disassembly of the nuclear envelope and lamina during mitosis in the early (<30-cell stage) C. elegans embryo (Lee et al., 2000), and that the maintenance of these nuclear structures until early anaphase may somehow limit and direct the movement of chromatin.

The second observation we made is that the chromatin masses undergo a reproducible rotation starting in late anaphase during the division of the zygote. Imaging of the $P$ granules and the second polar body revealed that it is the entire embryo which is rotating inside the egg shell. The sense of the rotation along the anterior-posterior axis was found to always be the same. Similar observations were made in a recent work using cortical granules and NMY-2-GFP as fiducial markers (Schonegg et al., 2014). The authors of this study took the invariant sense of the rotation to indicate the intrinsic chirality of components within the one-cell embryo. They further suggested that this chirality could provide cues to establish left-right asymmetry at later stages. Interestingly, whereas the cortical granules were observed to rotate by an angle of $\sim 120^{\circ}$, the nascent nuclei were found to do so by approximately half a turn $\left(180^{\circ}\right)$. This discrepancy remains to be explained; the possibility of differential rotation of organelles within the embryo deserves to be explored, in particular with regard to the nucleus, as it could represent a mean to change their cytoplasmic environment.

In summary, we have imaged the distribution of paternal and maternal chromatin relative to each other in the early living $C$. elegans embryonic nuclei. We found that intermingling of the parental chromatin first occurs in what we suggest is a brief G1 phase in the blastomeres of the 4-cell embryo. The Dendra2H2B-expressing worm strain that we generated should prove useful in future studies on cell lineages, chromatin mobility, and histone dynamics.

\section{Materials and Methods}

\section{Construction of a Dendra2-H2B expression cassette and insertion into a MosSCl vector}

A plasmid comprising an 852 bp synthetic Dendra2 sequence that has been codon-optimized for expression in C.elegans (Fig. S2) and that contains 3 short introns was obtained from IDT-DNA (lowa, USA). This sequence was PCR amplified using AttB-containing primers (forward 5'-ggggacaagtttgtacaaaaaagcaggctcaaaaatgaacaccccaggaatcaacc-3'; reverse 5'-ggggaccactttgtacaagaaagctgggtttacccgggccaaacttgggatgcaagtgg-3') and cloned in the Gateway pDONR221 plasmid using the BP Clonase enzyme mix (Life Technologies, CA, USA). The resulting plasmid is called CL31. A 386 bp fragment encoding a histone H2B (his-66) was amplified from C. elegans genomic DNAusing primers 5'-tcagctgcaggaggttctatgccaccaaagccatctgcc-3' (forward) and 5'-cttgctggaagtgtacttggtaacggc-3' (reverse) and cloned in-frame at the C-terminus of Dendra2 in the unique Smal site of CL31. The resulting plasmid is called CL33. The Gateway donor vectors containing Ce-Dendra2(CL31) or Ce-Dendra2-his-66(CL33) were sequence verified. CL33 was included along with pCFJ150 (a gift from Erik Jorgensen; Addgene \# 19329; (Frokjaer-Jensen et al., 2008)), pCM1.36 (a gift from Geraldine Seydoux;Addgene \# 17249) and pJA252 (a gift from Julie Ahringer; Addgene \# 21512; (Zeiser et al., 2011)) in a 4-way recombination reaction catalyzed by LR Clonase II Plus (Life technologies, CA, USA) in order to generate a Pmex-5::Dendra2::his-66::tbb-2 3' expression cassette flanked by the unc119 positive selection marker and left and right recombination arms for Mos 1 site ttTi5605 on chromosome II.

\section{Generation of worm strains by Mos1-mediated single copy insertion (MosSCI)}

C. elegans was maintained according to standard procedures (Stiernagle, 2006). Transgenic worm lines were generated using the MosSCI transgenesis method according to published protocols (http://www.wormbuilder.org/). Briefly, the expression vectors for 1) Dendra2-H2B (25 ng/ $\mu l)$; 2) the Mos recombinase (pCFJ601, $50 \mathrm{ng} / \mu \mathrm{l}$, Addgene \# 34874); 3) the co-injection markers (pCFJ90, $1.5 \mathrm{ng} / \mu \mathrm{l}$, Addgene \# 19327; pCFJ104, $7.8 \mathrm{ng} / \mathrm{\mu l}$, Addgene \# 19328; pGH8, $7.2 \mathrm{ng} / \mathrm{\mu l}$, Addgene \# 19359); and 4) a heat shock-inducible negative selection marker (pMA122, $10 \mathrm{ng} / \mu \mathrm{l}$, Addgene \# 34873) were microinjected in L4 and young adult Unc- worms from the EG6699 Mos1 insertion strain. All Addgene plasmids were gifts from Erik Jorgensen (Frokjaer-Jensen et al., 2008). After injection, worms were maintained at $25^{\circ} \mathrm{C}$ on OP50. Heat-shock was performed at $34^{\circ} \mathrm{C}$ for 2 hours $\sim 10$ days after injection. Unc+Cherry- worms were screened for the presence of a Dendra2-H2B signal in the gonads on an inverted microscope. The resulting transgenic strain was extensively characterized by PCR to ascertain insertion site. Homozygotes arose spontaneously and were backcrossed twice with the wild type N2 strain. The resulting strain is referred to as JBL1 [Pmex-5::Dendra2::his-66::tbb-2 3'UTR]. JBL1 was crossed with TH27 [tbg-1::gfp] or JH2108 [Pie-1p::gfp::pgl-1::pgl-13'UTR] to obtain strains JBL2 and JBL3, respectively.

\section{Imaging of Dendra2-H2B-expressing worms by selective plane illumination microscopy}

Adult worms were picked and transferred to $10 \mu \mathrm{l}$ of $0.5 \mathrm{mM}$ levamisole in M9 buffer. Two hundreds $\mu \mathrm{l}$ of $1 \%$ prewarmed low-melting point agarose was added. The resulting solution was mixed, aspirated into a glass capillary and left to solidify. The capillary was mounted in the chamber of a Zeiss Z.1 light sheet microscope filled with water. Imaging was done at room temperature with a 20X water objective (NA 1.0). The long axis of the worm was approximately parallel to the light sheet and optical sections $(\sim 80$ in total) were acquired with a z-step of $2 \mu \mathrm{m}$.

\section{Live imaging of early embryos, photoconversion, image processing}

Embryos were dissected in Early Embryo Dissection solution (EED: $4 \%$ sucrose in $0.1 \mathrm{M} \mathrm{NaCl}$ ) and mounted on an agarose pad ( $2 \%$ in EED). Samples were then covered with an $18 \mathrm{~mm} \times 18 \mathrm{~mm}$ coverslip (thickness $170 \pm 5 \mu \mathrm{m}$ ), which was fixed to the microscope slide with candle wax. Mineral oil was pipetted around the agarose pad to prevent drying of the embryos. Imaging was done at room temperature on an Olympus IX81 inverted microscope equipped with an Andor Revolution spinning disc system and a FRAPPA module (Andor, Belfast, United Kingdom). Samples were viewed with a 100X oil immersion objective (NA 1.40). Excitation light at $488 \mathrm{~nm}$ or $561 \mathrm{~nm}$ was provided by diode lasers set at powers of 5-7.5 mW (10-15\% of maximum power) and $10-12.5 \mathrm{~mW}$ (20-25\% of maximum power), respectively. Three dimensional stacks of images (xy pixel size of $133 \mathrm{~nm}$ ) were acquired with a z-spacing of $0.5 \mu \mathrm{m}$. Exposure to an Andor iXon Ultra 897 EMCCD camera lasted from 150-250 ms per optical section. Photoconversion was achieved in a region-of-interest (typical size of $\sim 5 \mu \mathrm{m} \times 5 \mu \mathrm{m})$ with the $405 \mathrm{~nm}$ diode laser set at a power of $4 \mathrm{~mW}(4 \%$ of maximum power) and a pixel dwell time of $40 \mu$ s for 5 repeats. Image stacks were processed, pseudo-colored and merged using ImageJ (http:// imagej.nih.gov/ij/index.html). The distances between the two centrosomes were calculated from the $x y z$ coordinates of the intensity centers of the $\gamma$-tubulin-GFP signals. The distance was set at $0 \mu \mathrm{m}$ when only one signal was detected. The extent of chromatin intermingling was assessed visually, based on the volume of the 'red' domain, i.e. the domains were considered to be spatially separated if the photoconverted chromatin occupied half of the nuclear volume or less. Otherwise, the domains were considered to 


\section{J. Bolková and C. Lanctôt}

be intermingled. Three dimensional reconstructions of image stacks were performed using the Amira_6.0 software from FEl.

\section{Acknowledgements}

We thank Zdeněk Švindrych and Guy Hagen for help with microscopy. We gratefully acknowledge the financial support of the Czech Science Foundation (grants P305/12/1246 and P302/12/G157). This work was also partially supported by Charles University in Prague (PRVOUK P27) LF1/1). The Imaging Center at our institute is supported by the European Regional Development Fund (OPPK CZ.2.16/3.1.00/24010). Some worm strains were provided by the CGC, which is funded by the US NIH Office of Research Infrastructure Programs (P40 OD010440).

\section{References}

BAO Z, ZHAO Z, BOYLE T J, MURRAY J I and WATERSTON R H (2008). Control of cell cycle timing during $C$. elegans embryogenesis. Dev Biol 318: 65-72.

BENENATIG, PENKOV S, MÜLLER-REICHERTT, ENTCHEV EV and KURZCHALIA T V (2009). Two cytochrome P450s in Caenorhabditis elegans are essential for the organization of eggshell, correct execution of meiosis and the polarization of embryo. Mech Dev 126: 382-393.

CHU D S, LIU H, NIX P, WU T F, RALSTON E J, YATES J R 3RD and MEYER B J (2006). Sperm chromatin proteomics identifies evolutionarily conserved fertility factors. Nature 443: 101-105.

DEPPE U, SCHIERENBERG E, COLE T, KRIEG C, SCHMITT D, YODER B and VON EHRENSTEIN G (1978). Cell lineages of the embryo of the nematode Caenorhabditis elegans. Proc Natl Acad Sci USA 75: 376-380.

EDGAR L G and MCGHEE J D (1988). DNA synthesis and the control of embryonic gene expression in C. elegans. Cell 53: 589-599.

FROKJAER-JENSEN C, DAVIS M W, HOPKINS C E, NEWMAN B J, THUMMEL J M, OLESEN S P, GRUNNET M and JORGENSEN E M (2008). Single-copy insertion of transgenes in Caenorhabditis elegans. Nat Genet 40: 1375-1383.

GALLO C M, WANG J T, MOTEGI F and SEYDOUX G (2010). Cytoplasmic partitioning of $\mathrm{P}$ granule components is not required to specify the germline in $C$. elegans. Science 330: 1685-1689.

GORJÁNÁCZ M and MATTAJ I W (2009). Lipin is required for efficient breakdown of the nuclear envelope in Caenorhabditis elegans. J Cell Sci 122: 1963-1969.

GURSKAYA N G, VERKHUSHA V V, SHCHEGLOV A S, STAROVEROV D B, CHEPURNYKH T V, FRADKOV A F, LUKYANOV S and LUKYANOV K A (2006). Engineering of a monomeric green-to-red photoactivatable fluorescent protein induced by blue light. Nat Biotechnol 24: 461-465.

ITO K and MCGHEE J D (1987). Parental DNA strands segregate randomly during embryonic development of Caenorhabditis elegans. Cell 49: 329-336.

ITO K, MCGHEE J D and SCHULTZ G A (1988). Paternal DNA strands segregate to both trophectoderm and inner cell mass of the developing mouse embryo. Genes Dev 2: 929-936.
KIMURA H and COOK P R (2001). Kinetics of core histones in living human cells: little exchange of $\mathrm{H} 3$ and $\mathrm{H} 4$ and some rapid exchange of $\mathrm{H} 2 \mathrm{~B}$. J Cell Biol 153: 1341-1353.

KIPREOS E T (2005). C. elegans cell cycles: invariance and stem cell divisions. Nat Rev Mol Cell Biol 6: 766-776.

LEE K K, GRUENBAUM Y, SPANN P, LIU J and WILSON K L (2000). C. elegans nuclear envelope proteins emerin, MAN1, lamin, and nucleoporins reveal unique timing of nuclear envelope breakdown during mitosis. Mol Biol Cell11:3089-3099.

MAYER W, NIVELEAU A, WALTER J, FUNDELE R and HAAF T (2000a). Demethylation of the zygotic paternal genome. Nature 403: 501-502.

MAYER W, SMITH A, FUNDELE R and HAAF T (2000b). Spatial separation of parental genomes in preimplantation mouse embryos. J Cell Biol 148: 629-634.

OOI S L, PRIESS J R and HENIKOFF S (2006). Histone H3.3 variant dynamics in the germline of Caenorhabditis elegans. PLoS Genet 2: e97.

OSWALD J, ENGEMANN S, LANE N, MAYER W, OLEK A, FUNDELE R, DEAN W, REIK W and WALTER J (2000). Active demethylation of the paternal genome in the mouse zygote. Curr Biol 10: 475-478.

SAMSON M, JOW M M, WONG C C, FITZPATRICK C, ASLANIAN A, SAUCEDO I, ESTRADA R, ITO T, PARK S K, YATES J R 3RD et al., (2014). The specification and global reprogramming of histone epigenetic marks during gamete formation and early embryo development in C. elegans. PLoS Genet 10: e1004588.

SANTOS F, HENDRICH B, REIK W and DEAN W (2002). Dynamic reprogramming of DNA methylation in the early mouse embryo. Dev Biol 241: 172-182.

SCHONEGG S, HYMAN AA and WOOD W B (2014). Timing and mechanism of the initial cue establishing handed left-right asymmetry in Caenorhabditis elegans embryos. Genesis 52: 572-580.

SINGH D and POHL C (2014). Coupling of rotational cortical flow, asymmetric midbody positioning, and spindle rotation mediates dorsoventral axis formation in $C$ elegans. Dev Cell 28: 253-267.

STIERNAGLET (2006). Maintenance of C. elegans. In WormBook, (Ed. COMMUNITY, T. C. E. R.), vol. doi/10.1895/wormbook.1.101.1, pp.1-11.

STRICKFADEN H, ZUNHAMMER A, VAN KONINGSBRUGGEN S, KÖHLER D and CREMER T (2010). 4D chromatin dynamics in cycling cells: Theodor Boveri's hypotheses revisited. Nucleus 1: 284-297.

SULSTON J E, SCHIERENBERG E, WHITE J G and THOMSON J N (1983). The embryonic cell lineage of the nematode Caenorhabditis elegans. DevBiol100:64-119.

THOMSON I, GILCHRIST S, BICKMORE W A and CHUBB J R (2004). The radial positioning of chromatin is not inherited through mitosis but is established de novo in early G1. Curr Biol 14: 166-172.

WALTER J, SCHERMELLEH L, CREMER M, TASHIRO S and CREMER T (2003) Chromosome order in HeLa cells changes during mitosis and early $\mathrm{G} 1$, but is stably maintained during subsequent interphase stages. J Cell Biol 160: 685-697.

ZEISERE, FROKJAER-JENSEN C, JORGENSENE and AHRINGERJ (2011). MosSC and Gateway compatible plasmid toolkit for constitutive and inducible expression of transgenes in the C. elegans germline. PLoS One 6: e20082. 


\section{Further Related Reading, published previously in the Int. J. Dev. Biol.}

A conserved set of maternal genes? Insights from a molluscan transcriptome

M. Maureen Liu, John W. Davey, Daniel J. Jackson, Mark L. Blaxter and Angus Davison

Int. J. Dev. Biol. (2014) 58: 501-511

http://dx.doi.org/10.1387/ijdb.140121ad

Interplay between DNA methylation, histone modification and chromatin remodeling in stem cells and during development

Kohta Ikegami, Jun Ohgane, Satoshi Tanaka, Shintaro Yagi, and Kunio Shiota

Int. J. Dev. Biol. (2009) 53: 203-214

http://dx.doi.org/10.1387/ijdb.082741ki

Differences in embryonic pattern formation between Caenorhabditis elegans and its close parthenogenetic relative Diploscapter coronatus

Vera Lahl, Jens Schulze and Einhard Schierenberg

Int. J. Dev. Biol. (2009) 53: 507-515

http://dx.doi.org/10.1387/ijdb.082718vl

Histone deacetylase 1 and 2-controlled embryonic development and cell differentiation Reinhard Brunmeir, Sabine Lagger and Christian Seiser

Int. J. Dev. Biol. (2009) 53: 275-289

http://dx.doi.org/10.1387/ijdb.082649rb

Dynamic alterations of linker histone variants during development

James S. Godde and Kiyoe Ura

Int. J. Dev. Biol. (2009) 53: 215-224

http://dx.doi.org/10.1387/ijdb.082644jg

Epigenetic reprogramming of the genome--from the germ line to the embryo and back again

K L Arney, S Erhardt, R A Drewell and M A Surani

Int. J. Dev. Biol. (2001) 45: 533-540

http://dx.doi.org/10.1387/ijdb.11417896

5 yr ISI Impact Factor $(2013)=2.879$
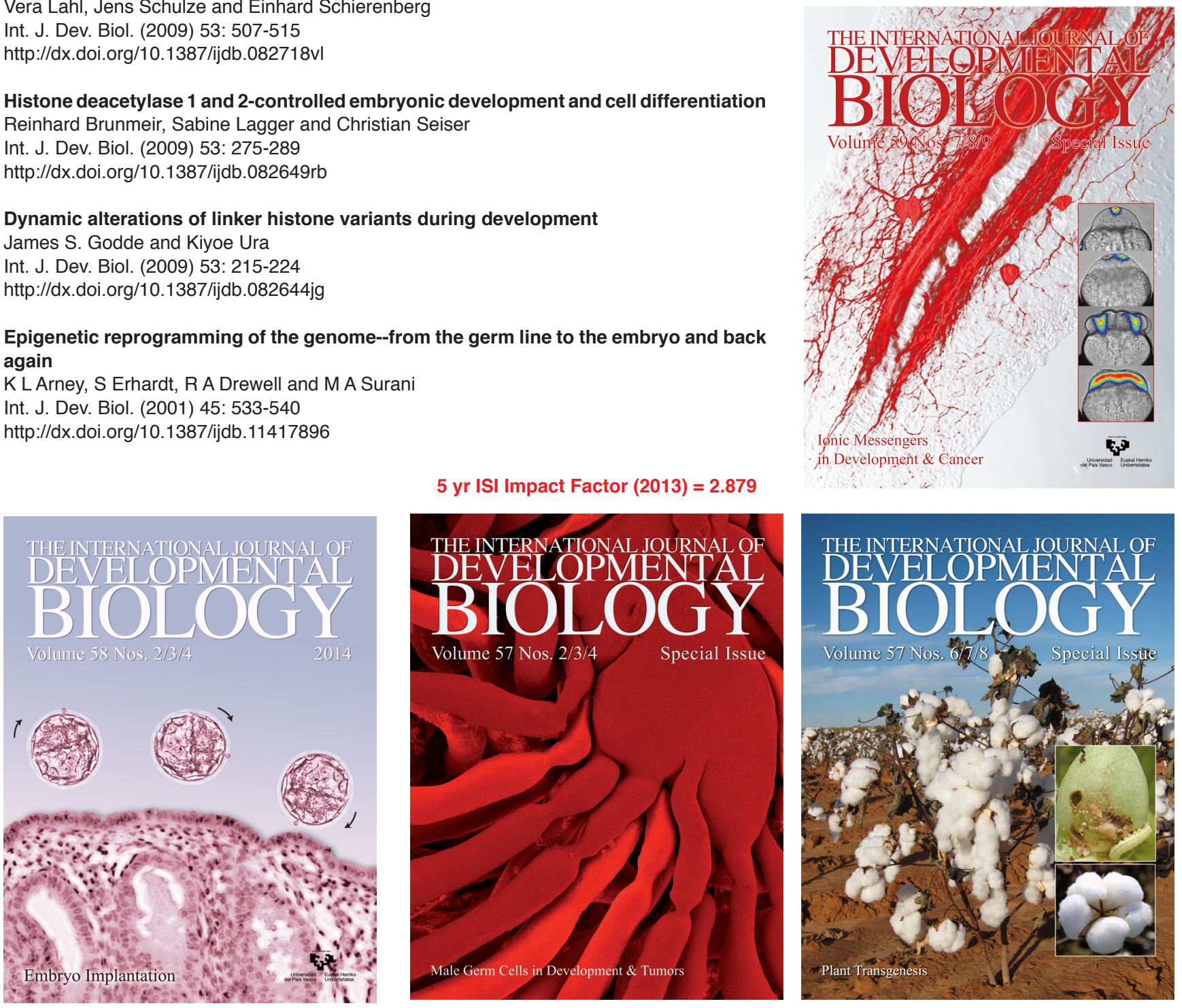